\title{
Decolorization Potential and Comparative Laccase Activity of Pleurotus Species
}

\author{
Suman Kumar", R. K. Singh and Pragya Bharti \\ Department of mycology \& plant Pathology, Institute of Agricultural Sciences, Banaras Hindu \\ University, Varanasi - 221005, Utter Pradesh, India \\ *Corresponding author
}

\section{A B S T R A C T}

\section{Keywords}

Pleurotus spp., Decolorization, Synthetic dyes, Laccase activity

Article Info

Accepted:

18 July 2020

Available Online:

10 August 2020
Pleurotus spp which are already widely known for its nutrient values, four of them were collected viz., P. ostreatus, $P$. florida, P. sajor-caju, $P$. flabellatus and studies were made to detect their efficiency in terms of laccase enzyme production and dye decolorization efficiency for its industrial uses. Bromophenol blue, crystal violet, methyl red, methylene blue and brilliant blue were the five dyes mostly used on the commercial side and thus were nominated for this study on potato dextrose agar (solid medium) and potato dextrose broth (liquid medium) at three different concentrations i.e. 100ppm, 200ppm and 500ppm. The outcome at a gap of 4 days were noted down and proved Pleurotus florida as the most promising strain for decolorization of all the above mentioned dyes, in solid (PDA) as well as liquid broth media due to their lignolytic enzymes. $P$. sajor-caju and $P$. ostreatus also found to be effective for dye decolorization on solid medium as well as on liquid culture condition. P. ostreatus (96\%) was found best for decolorization of bromophenol blue on solid medium and $P$. florida $(76.33 \%)$ on liquid medium was best, for decolorization of crystal violet. Laccase activity was found to be maximum for $P$. florida (720.3U) during initial period and the production of laccase decreased with time, while it was reverse in case of $P$. ostreatus where laccase activity increased with increase in incubation periods. This study verifies the fungus to act as a biological agent to degrade synthetic dyes and for its better commercial uses.

\section{Introduction}

The eukaryotic organism called fungi having wide applications in agricultural, botanical, biotechnological and medical fields are also used as food from elderly times in form of mushroom, morels, truffles and mycoprotein. Mushrooms are better known and proficiently used for nutritional purposes especially
Pleurotus spp (Oyster mushroom), including Plerotus ostreatus, Pleurotus florida, Pleurotus sajor-cajuetc, Agaricus bisporus (button mushroom), Calocybe indica (milky white mushroom) adding to other significant properties such as nematode control, synthetic dye decolorization, biodegradation (Smith et. al., 2002). The practice of mushroom cultivation was initially flourishing for its 
nutritional and medicinal value. But the boost to the industry was seen when the same mushroom was found to possess the ability to degrade plastic, plastic waste or synthetic dyes based on its enzymatic activity mainly laccase which has created havoc in recent times. The biodegradable polymers can be converted into methane, humus, carbon dioxide, water, biomass and other substances, the catalytic role therein played by these Pleurotus spp.

The colored organic or inorganic substance which has an affinity to the substrate based on the chemical bonds or the chemical reaction are known as dyes. The main constituent groups of synthetic dyes are chromophore responsible for dye color due to saturation and auxochrome group responsible for dye fiber reaction. Synthetic dyes can be acidic or basic in their chemical reaction, acid dyes containing one or other sulphonic acid constituent are water soluble, with high anionic ratio, fixation of dye with fibre by formation of salt between cations in the fiber and anions in the dye, used for nitrogenous group containing fibers and silk, commonly used acidic dyes are triphenyl, azo and anthriquinone. Basic/ Cationic dye made of coal tar derivatives, commercially used on acrylic fiber and some are used for wools, silk, polyester and paper printing such as Basic brown 1. As the uses of dye in industries are rapidly increasing the methods and solutions to disintegrate the dyes are also getting importance.

Wastewater containing toxic dyes released from textile and other industries have to be treated due to the tremendous hazards and public concern about their toxicity and carcinogenicity. Physical and physiochemical methods to remove the dyes from industrial waste such as flocculation, oxidation, filtration is neither affordable nor practically possible. Not only physical but also some chemical methods are also used for dye decolorization such as oxidation, adsorption and electrolysis which are expensive. Now the latest research is focused on decolorization of dyes by biological methods involving microorganisms and enzymes. Replacing the conventional treatment technologies with more practical, environment friendly techniques using microorganisms is the call of recent times (Hamman, 2004).

Thus to add some information in the field of biodegradation we introduce this study where we compare the decolorization potential of different Pleurotus spp. viz., P. ostreatus, $P$. sajor-caju, $P$. floridaand $P$. flabellatus and there Laccase enzyme activity under solid and broth culture condition.

\section{Material and Methods}

\section{Collection of culture and their maintenance}

Different species of Pleurotus viz., P. sajorcaju, $P$. florida, $P$. flabellatus and $P$. ostreatus were obtained from the Mushroom production unit Department of Mycology and Plant Pathology, Institute of Agricultural Sciences, Banaras Hindu University (BHU), Varanasi. The cultures were maintained on Potato Dextrose Agar (PDA) slants and plates were incubated at $25 \pm 2{ }^{\circ} \mathrm{C}$ and regularly used for experiment.

\section{Dyes preparation}

Required quantity of different dyes were measured by using electronic balance and concentration of $5000 \mathrm{ppm}$ stock solution of Bromophenol blue, Crystal violet, Methyl red, Methylene blue and Brilliant blue dyes prepared with sterilized distilled water, measured quantity of dyes were dissolved in $100 \mathrm{ml}$ distilled water, and heated for proper dissolution of dye into water For the preparation of 5000 ppm stock solution 500 
$\mathrm{mg}$ of each dye was taken and dissolved in $100 \mathrm{ml}$ of distilled water to get desired concentration of stock solution. For estimation of Decolorization potential of Pleurotus spp three different concentrations (100ppm, 200ppm, 500ppm) of each five dyes were taken (Pointing 2001).

\section{Decolorization assay}

Five synthetic dyes that are commonly used in textile and coloring industries i.e. Methyl red, Bromophenol blue, Methylene blue, Crystal violet, and Brilliant blue were studied. The decolorization efficiency of different Pleurotus spp. namely $P$. florida, $P$. flabellatus, $P$. sajor-caju and $P$. ostreatus were checked against these dyes at varying concentration and period of incubation.

\section{Qualitative assay}

Culture medium supplemented with three different concentration i.e. 500 ppm, 200 ppm and $100 \mathrm{ppm}$ of each dyes viz. Bromophenol blue, Crystal violet, Methyl red, Methylene blue and Brilliant blue were poured into 90 $\mathrm{mm}$ Petri plates and allowed to solidify under Laminar cabinet (Radhika et al., 2014). The plates were inoculated with $5 \mathrm{~mm}$ mycelial disc from 7 days old pure culture and the positive control were used as unsupplemented Agar plates inoculated with respective culture and negative control were used as the un-inoculated plates supplemented with respective dye concentration under laminar air flow chamber and incubated at $25 \pm 2^{\circ} \mathrm{C}$. Observations were recorded at 4 days interval till $16^{\text {th }}$ day of incubation by measuring the diameter of mycelial growth as well as decolorized zone produced by different Pleurotus spp.

\section{Quantitative assay}

Potato Dextrose broth (PDB) were prepared and poured in Erlenmeyer flasks of $150 \mathrm{ml}$ capacity. These flasks were poured with 50 $\mathrm{ml}$ potato dextrose broth and sterilized by autoclaving at $121^{\circ} \mathrm{C}$ for 20 minutes at $15 \mathrm{psi}$ pressure. These sterilized flasks were inoculated with respective culture of Pleurotus spp, and incubated for 6 days at $25 \pm 2^{\circ} \mathrm{C}$. After 6 days of incubation already inoculated flasks were supplemented with 100ppm, 200 ppm and 500 ppm concentration of respective dyes from prepared stock solution and kept in incubator at $25 \pm 2{ }^{\circ} \mathrm{C}$. Absorbance of dye supplemented broth were recorded at 4 days interval till $16^{\text {th }}$ day by using UV-spectrophotometer at their respective wavelength $590 \mathrm{~nm}$ for Bromophenol blue, $590 \mathrm{~nm}$ for Crystal violet, $540 \mathrm{~nm}$ for Methyl red, $660 \mathrm{~nm}$ for Methylene blue and $595 \mathrm{~nm}$ for Brilliant blue dyes.

The quantification of dye decolorization and dye decolorization efficiency of different Pleurotus spp. was done by using Formula:

Decolorization percentage $(\%)=\frac{\text { Initial0D-Final0D }}{\text { Initial0D }} \times 100$

\section{Laccase enzyme assay}

The activity of laccase enzyme in the media was explained briefly by Turner (1974) for the first time and slight changes were made by Singh et al., (1988). Qualitative laccase enzyme assay was done by Agar plate method where Pleurotus species cultured on PDA plates supplemented with guaiacol $(0.04 \%)$ and chloramphenicol $(0.01 \%)$ and after 72 hours, reddish brown zones of fungal colonies were visually observed.

For quantitative laccase enzyme assay by Guaiacol enzyme extract method was used where $50 \mathrm{ml}$ PDB/ flask was taken for the growth of the fungus. The enzyme activity in broth was quantitatively analysed by taking absorbance by spectrophotometer at regular interval of $4^{\text {th }}$ day, $8^{\text {th }}$ day, $12^{\text {th }}$ day and $16^{\text {th }}$ day of incubation. The reaction mixture was 
prepared by dissolving Guaiacol $2 \mathrm{mM}$, $14.214 \mu \mathrm{l}$ of extra pure guaiacol into $50 \mathrm{ml}$ of sterilized distilled water, Sodium acetate buffer of $10 \mathrm{mM}, 5.5 \mathrm{pH}$ was prepared by using $0.218 \%(10.9 \mu \mathrm{l})$ acetic acid and $0.871 \%(43.55 \mu \mathrm{l})$ of sodium acetate in $50 \mathrm{ml}$ of distilled water. the amount of Guaiacol (2 $\mathrm{mM})$ of $1 \mathrm{ml}$ and sodium acetate buffer (10 $\mathrm{mM}$ ) of $3 \mathrm{ml}$ was added in the $1 \mathrm{ml}$ of filtered supernatant and incubated for $20 \mathrm{~min}$ at $30^{\circ} \mathrm{C}$, and the color change was observed i.e. reddish brown color of the whole reaction mixture observed due to the oxidation of guaiacol by the laccase enzyme. The absorbance of the reaction mixture at $450 \mathrm{~nm}$ was recorded using an UVspectrophotometer. The enzyme activity is expressed in the form of International Units (IU). $1 \mathrm{IU}$ is the amount of enzyme which is consumed to oxidize $1 \mu \mathrm{mol}$ of guaiacol per min was calculated by using the formula:

$E . A=A \frac{V}{t} \times e \times v$

E.A = Enzyme activity, $\mathrm{A}=$ Absorbance, $\mathrm{V}$ $=$ Total mixture volume $(\mathrm{ml}), \mathrm{v}=$ enzyme volume $(\mathrm{ml}), \mathrm{t}=$ incubation time, Extinction coefficient for guaiacol $(0.6740 \mu \mathrm{M} / \mathrm{cm})$ (Kalra et al., 2013).

\section{Results and Discussion}

\section{Effect of synthetic dyes concentration on mycelial growth of Pleurotus spp.}

The effect of synthetic dyes concentrations on the mycelial growth of different Pleurotus spp. is depictured in Fig. 1. Diameter of mycelial growth of each Pleurotus spp. at day 16 are represented in Fig. 1. Growth of all Pleurotus spp. were negatively affected with an increasing concentration of dyes in culture medium. Almost complete growth inhibition was reported with crystal violet $(500 \mathrm{ppm})$ and $P$. sajor-caju was found most efficient with respect of mycelial growth on dye supplemented growth media. Ramsay and Nguyan (2002) reported similar finding with Amaranth dye. It has been reported that a more toxic effect of Amaranth dye at higher concentration of $100 \mathrm{mg}$ of dye in kirk's basal salt medium containing $1 \mathrm{~g}$ of glucose concentration on growth of $\mathrm{T}$. versicolor then a lower dye concentration of $33 \mathrm{mg}$ in same culture condition.

\section{Effect of synthetic dyes concentration on Decolorization efficiency of Pleurotus spp.}

It was observed that most of the Pleurotus species were more efficient at 100 and 200 ppm dyes concentrations and comparatively less efficient at higher dye concentration for decolorization. All the four strains showed differences in decolorized zone formation with respect to each other when being incubated with the same concentration of respective synthetic dyes. Maximum decolorization zone formation was reported for bromophenol blue dye by all four Pleurotus spp while least decolorized zone formation was observed for methylene blue and brilliant blue dyes (Fig. 2).

A significant decolorization of all the dyes by Pleurotus spp. was observed after 16 days of incubation. More than $70 \%$ decolorization were observed for bromophenol blue and crystal violet at $100 \mathrm{ppm}$ after 16 days of incubation under liquid culture condition. A $80.7 \%$ and $72 \%$ of decolorization of crystal violet was observed at $200 \mathrm{ppm}$ dye concentration after 16 days by $P$. flabellatus and $P$. florida respectively. A $67 \%$ decolorization after 16 days was observed for crystal violet by $P$. florida (Fig. 3). Similar results have been reported in literature in which three Pleurotus spp viz., P. florida, $P$. ostreatus $P$. sajor-cajuwere used to study their mycelial growth pattern and decolorized zone formation on PDA (Radhika et al., 
2014). Similar results were also reported by Jebapriya and Gnanadoss (2013), for decolorization of bromophenol blue by $P$. florida and other white rot fungi. The ability of three Pleurotus species (P. sajor-caju LCJ 184, P. florida LCJ 65, P. ostreatus LCJ 183) was screened for the decolorization of bromophenol blue using solid and liquid medium. All three Pleurotus species were found effective in decolorizing the dyes on potato dextrose agar plate but the decolorization efficiency varied for species to species and it was found that P. sajor-caju effectively decolorized the selected dyes by 85-98\%.

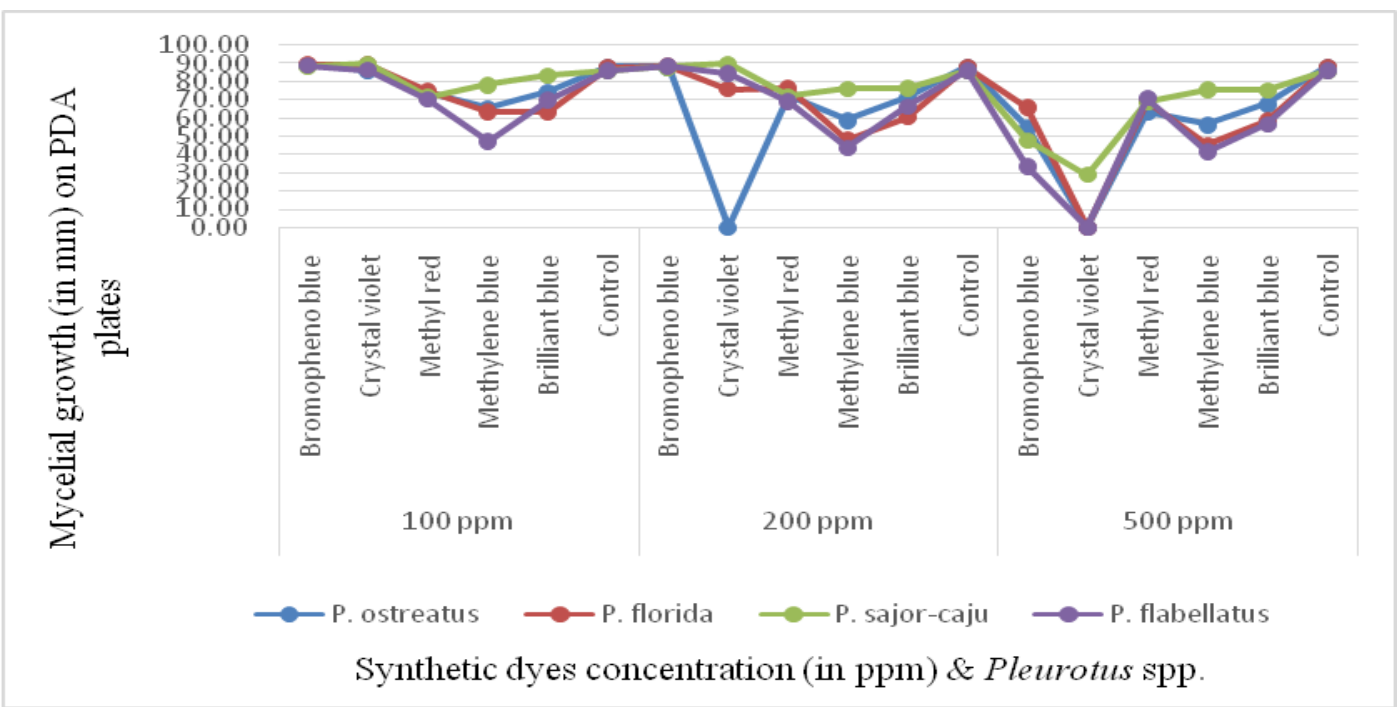

Fig.1 Radial growth of Pleurotus spp. (mm) on agar plates supplemented with different concentrations of respective dye

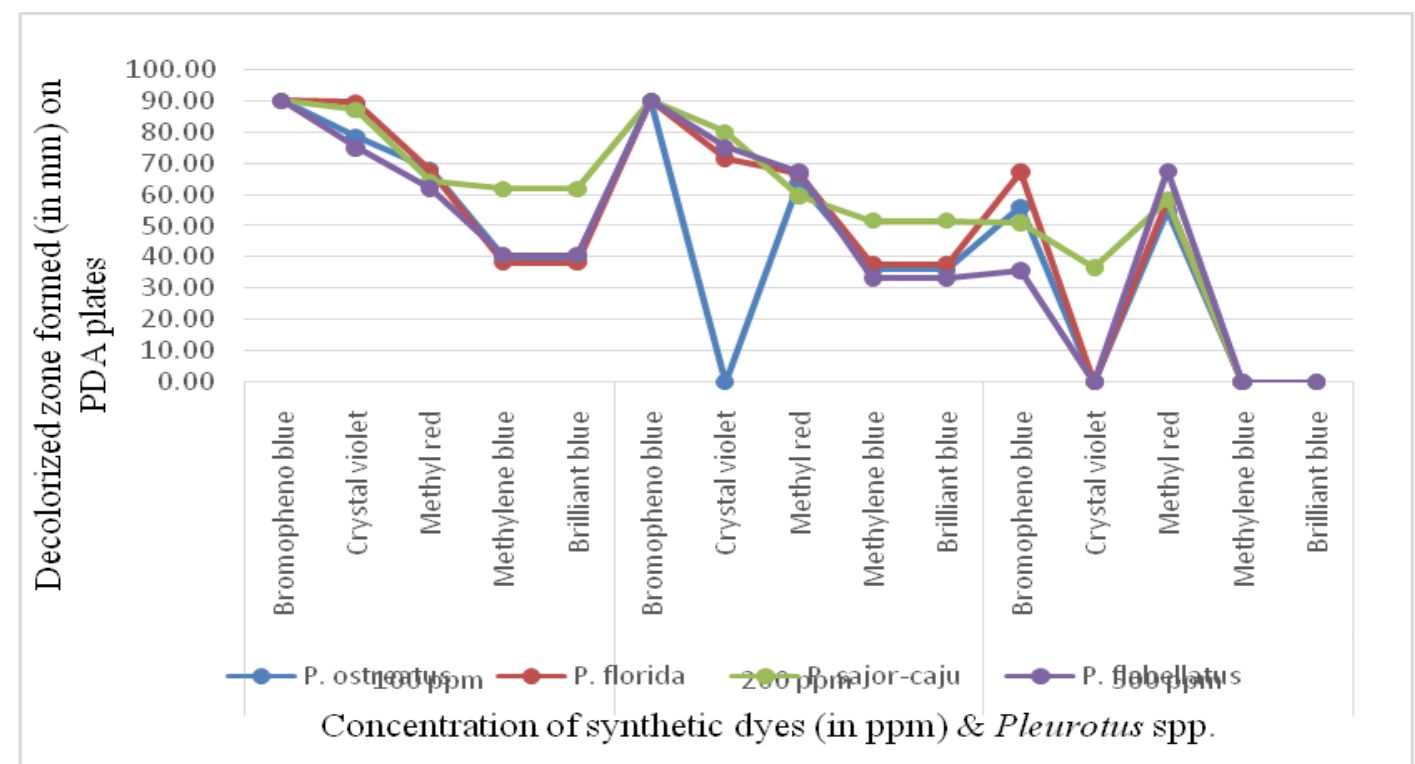

Fig.2 Decolorized zone formation by four Pleurotus spp at three different concentration of five respective synthetic dyes 


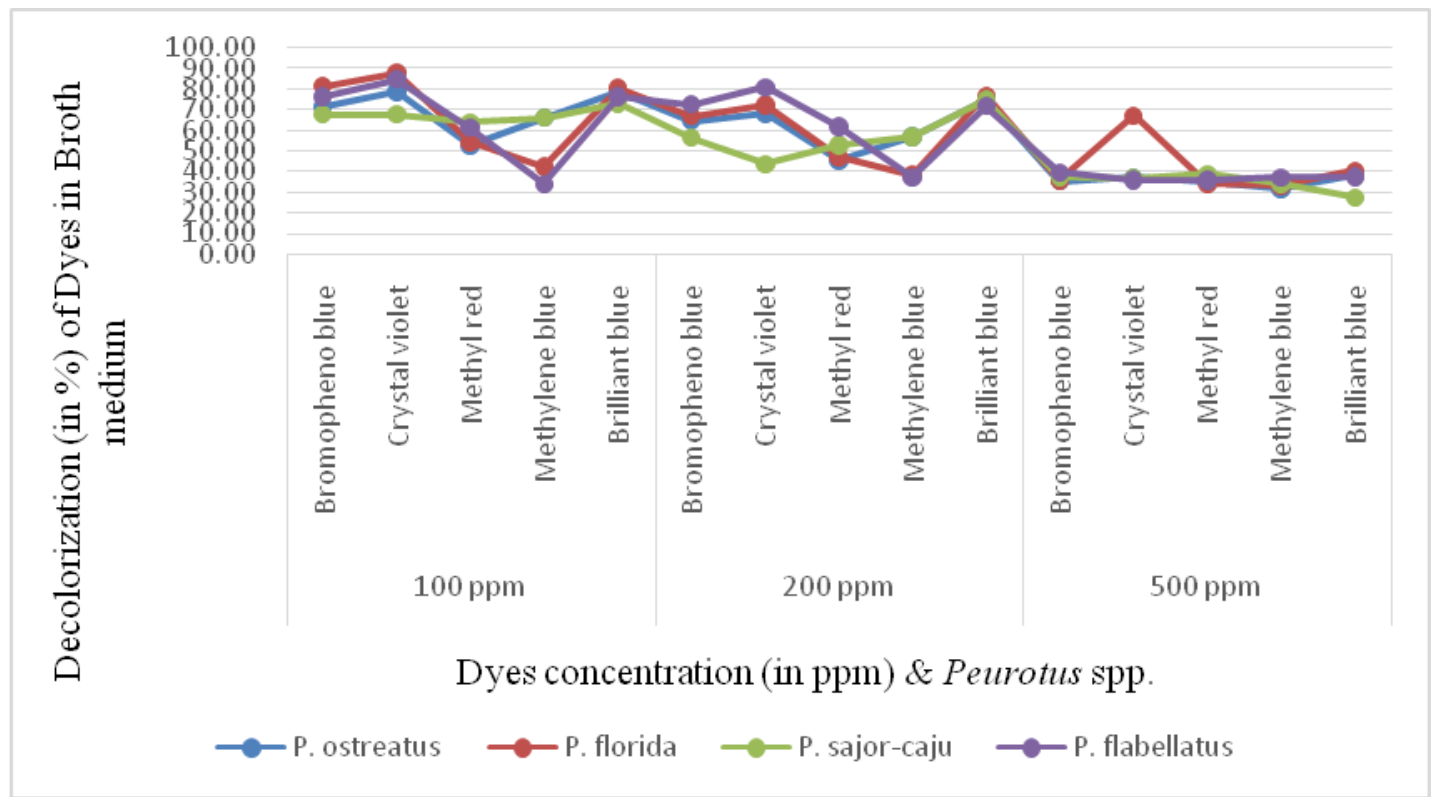

Fig.3 Decolorization (in \%) of all five synthetic dyes at three different concentrations by four Pleurotus species

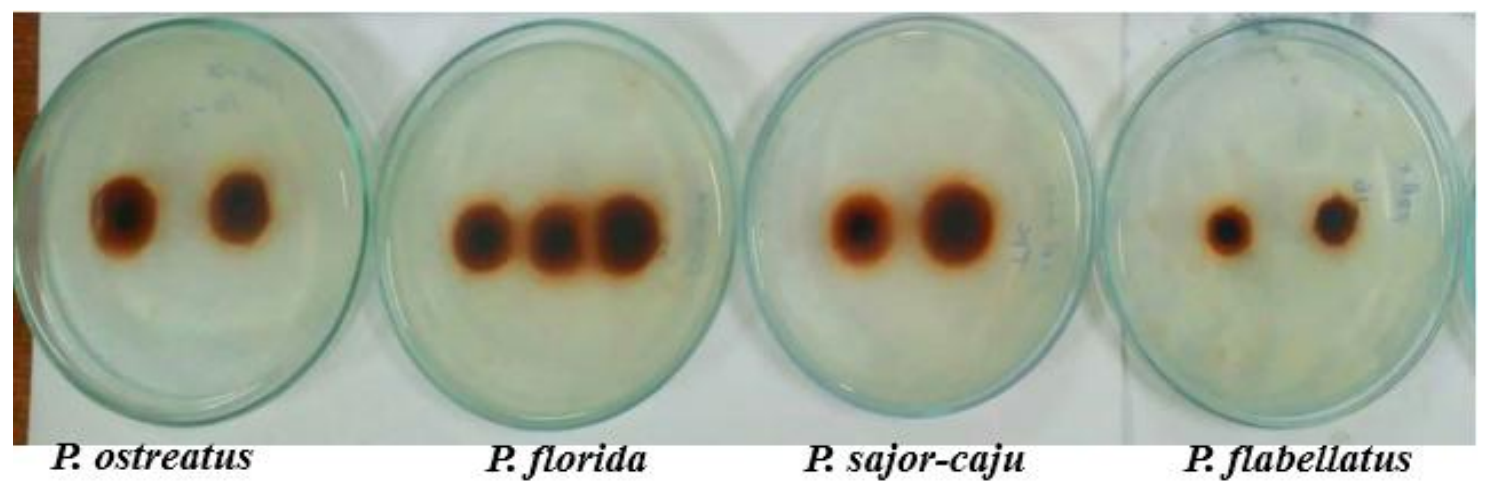

Fig.4 Reddish-brown colonies formed apparently due to laccase produced by Pleurotus spp. as a positive result on agar plate

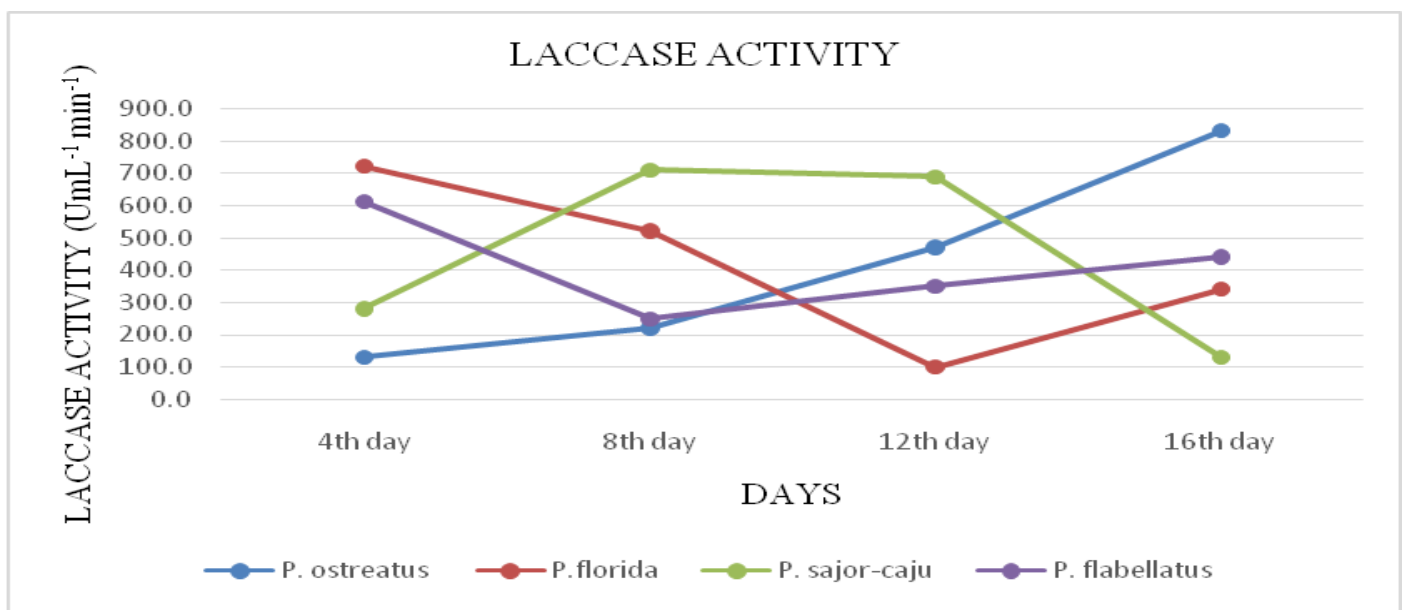

Fig.5 Extracellular laccase enzyme production at different growth stages of Pleurotuas spp 
Table.1 Laccase activity on agar plate after $72 \mathrm{~h}$ of incubation

\begin{tabular}{|l|l|l|l|l|}
\hline Enzyme & Laccase activity & \multicolumn{4}{l|}{} \\
\hline Strains & P. ostreatus & P. florida & P. sajor-caju & P. flabellatus \\
\hline Reactions & ++ & ++ & ++ & + \\
\hline
\end{tabular}

(+) small reddish brown zone (++) Dark reddish brown zone

\section{Laccase assay}

\section{Qualitative Laccase assay}

The qualitative laccase activity of Pleurotus spp. was studied on the agar plates supplemented with guaiacol $(0.04 \%)$ and chloramphenicol $(0.01 \%)$ to avoid bacterial contamination after 72 hours of incubation the results as observed are described in Table no. 3.3.1. When compared to $P$. flabellatus, which showed smaller colored zone while $P$. ostreatus, P. florida and P. sajor-caju, these three species showed better laccase activity comparatively. Similar findings were published by Nadeem et al., (2014), when he saw the formation of reddish brown zone due to oxidation of guaiacol and confirmed the presence of laccase. He made a conclusion that the quick color formation with guaiacol is easy and reliable source for laccase screening (Fig. 4).

\section{Quantitative Laccase assay}

Extracellular Laccase activity of all the Pleurotus species is presented in Figure 5. Among all four species after 4 days of growth $P$. florida (720.3U) achieved maximum followed by $P$. flabellatus (610.7U) Laccase activity. The Laccase activity of $P$. ostreatus increasing continuously with increase in growth periods and on $16^{\text {th }}$ days maximum extracellular enzyme (Laccase) activity was reported for $P$. ostreatus (830U) followed by P. flabellatus (440U). Similar results have been reported in literature in which $P$. florida strain achieved 425U extracellular enzyme activity whereas $P$. flabellatus and $P$. ostreatus showed 358U and 297.3U Laccase activity respectively after 5 daysand maximum Laccase activity reported in $P$. florida strain (725.2U) after 10 days (Singh and Srivastava, 2016).Tellez et al., (2008), reported similar results when $P$. ostreatus cultures grown in submerged fermentation producing laccase as $0.09 \mathrm{U} / \mathrm{ml}$. The results showed that $P$. ostreatus performs much better in submerged fermentation than in solid-state fermentation

In conclusion, all the four Pleurotus spp. viz. $P$. ostreatus, $P$. florida, $P$. sajor-caju, $P$. flabellatus were detected for their efficiency to decolorize five dyes namely bromophenol blue, crystal violet, methyl red, methylene blue, and brilliant blue at three different concentrations (100ppm, 200ppm, 500ppm) on potato dextrose agar (solid medium) and potato dextrose broth (liquid medium).

The decolorization of dyes and laccase activity in liquid medium was assessed by use of UV-spectrophotometer. It was observed that most of the Pleurotus strains were more efficient at 100 and 200 ppm concentrations and comparatively less efficient at higher dye concentration for decolorization, among which Pleurotus florida was observed as the most promising strain for decolori+9zation of all the above mentioned dyes, in solid (PDA) as well as liquid broth media. Laccase activity was found to be maximum for $P$. florida during initial period and the production of laccase decreased with time, while it was reverse in case of $P$. ostreatus where laccase activity increased with increase in incubation periods. 
The consistency in performance of Pleurotus species has to be evaluated and assured in order to realize the application of Pleurotus spp in the biodegradation and mycoremediation process in the near future and to be judiciously utilized for remediation of dye effluent released from industries at large scale.

\section{References}

Hamman, S. (2004). Bioremediation capabilities of white rot fungi. Biodegradation, 52(16), 11.

Jebapriya, G. R., and Gnanadoss, J. J. (2013). Bioremediation of textile dye using white rot fungi: A review. International Journal of Current Research and Review, 5(3), 1.

Kalra, K., Chauhan, R., Shavez, M., Sachdeva, S., (2013). Isolation oflaccase producing Trichoderma spp. and effect of $\mathrm{pH}$ and temperature on its activity. International Journal of Chemistry and Environmental Technology 5 (5), 2229-2235.

Nadeem, A., Baig, S., and Sheikh, N. (2014). Mycotechnological production of laccase by Pleurotus ostreatus $\mathrm{P} 1$ and its inhibition study. Journal of Animal and Plant Science, 24(2), 492-502.

Pointing, S. (2001). Feasibility of bioremediation by white-rot fungi. Applied microbiology and biotechnology, 57(1-2), 20-33
Radhika, R., Jebapriya, G. R., and Gnanadoss, J. J. (2014). Decolorization of synthetic textile dyes using the edible mushroom fungi Pleurotus. Pakistan Journal Biological Science, 17, 248-253.

Ramsay, J. A., and Nguyen, T. (2002). Decoloration of textile dyes by Trametes versicolor and its effect on dye toxicity. Biotechnology Letters, 24(21), 1757-1761.

Singh R P, Garcha H S and Khanna P K (1988) Laccase production by Pleurotus spp. Indian Journal of Microbiology 28: 38-41.

Singh, M. P., and Srivastava, A. K. (2016). Decolorization of synthetic textile dye and enzymes production by improved strains of Pleurotus species. Cell and Molecular Biology, 62(3).

Smith, M, Adam G, Duncan HJ, and Cowling MJ (2002) The effects of cationic surfactants on marine biofilm growth on hydrogels. Estuarine, Coastal and Shelf Science 55(3): 361-67.

Tellez-Tellez, M., Fernandez, F. J., MontielGonzalez, A. M., Sanchez, C., and Diaz-Godinez, G. (2008). Growth and laccase production by Pleurotus ostreatus in submerged and solid-state fermentation. Applied microbiology and biotechnology, 81(4), 675.

Turner, E. M. (1974). Phenoloxidase activity in relation to substrate and development stage in the mushroom, Agaricus bisporus. Transactions of the British Mycological Society, 63(3), 541-547.

\section{How to cite this article:}

Suman Kumar, R. K. Singh and Pragya Bharti. 2020. Decolorization Potential and Comparative Laccase Activity of Pleurotus Species. Int.J.Curr.Microbiol.App.Sci. 9(08): 1935-1942. doi: https://doi.org/10.20546/ijcmas.2020.908.221 